\title{
Effect of acute aerobic exercise in different times of day on iron status and hematological factors in professional football players
}

\author{
Majid Mardaniyan Ghahfarrokhi ${ }^{1{ }^{(\mathbb{D}}}$, Abdulhamid Habibi², Hamed Rezaei Nasab ${ }^{3}$ \\ ${ }^{1} \mathrm{PhD}$ Student of Exercise Physiology, Department of Sport Sciences, Shahrekord University, Shahrekord, Iran. \\ ${ }^{2}$ Professor of Exercise Physiology, Department of Exercise Physiology, Shahid Chamran University of Ahvaz, Ahvaz, Iran \\ ${ }^{3} \mathrm{PhD}$ Student of Exercise Physiology, Faculty of Sport Sciences, Shahid Chamran University of Ahvaz, Ahvaz, Iran
}

*Corresponding Author: Majid Mardaniyan Ghahfarrokhi, PhD Student of Exercise Physiology, Department of Sport Sciences, Shahrekord University, Shahrekord, Iran. Email: majid.mardaniyan@gmail.com

\begin{abstract}
Background and aims: Exercise time is one of the main challenges of athletes. The purpose of this study was to investigate the effect of acute aerobic exercise at different times of the day on iron status and hematological factors in professional football players.

Methods: In this quasi-experimental study, 40 professional football players were randomly divided into morning exercise, evening exercise, morning control, and evening control groups. The experimental groups performed Bruce's test in the morning and evening. To measure iron, ferritin, red blood cell (RBC), and hemoglobin ( $\mathrm{Hb}$ ), the blood samples were taken before, immediately after, as well as 24 and 72 hours after the Bruce test. Repeated-measure ANOVA and two-way ANOVA (group*time) were used to analyze the obtained data.

Results: The results showed a significant increase immediately after an acute aerobic exercise while demonstrating a significant decrease in $\mathrm{RBC}$ and $\mathrm{Hb}$ in morning and evening exercise groups 24 and 72 hours after the exercise compared to the control groups. In addition, a significant reduction immediately after the acute aerobic exercise, whereas a significant increase 24 and 72 hours after the exercise, was observed in the serum levels of iron and ferritin in the morning and evening exercise groups compared to the control groups. As regards the serum levels of iron, an immediate significant decrease, along with a significant increase 24 hours after the acute aerobic exercise was found between the morning and evening exercise groups $(P \leq 0.05)$.

Conclusion: It seems that performing acute endurance training in the evening is safe in terms of reducing the serum levels of iron and developing the "exercise-induced anemia". Finally, at least 24 hours is required for the recovery of hematological parameters after acute endurance training.

Keywords: Acute aerobic activity; Circadian rhythm; Iron status; Hematology factors; Professional football player
\end{abstract}

Received: 13 February 2018, Accepted: 12 June 2018, ePublished: 24 June 2019

\section{Introduction}

Physiological and hematological changes in athletes after training and competition have always been of interest to the sports researchers. In addition, the intensity, duration, and the type of exercise are factors that affect the performance, along with physiological and hematological changes in athletes (1). Training time has always been a challenge for improving performance and reducing the inflammation for the athletes. It is recognized that the best sport performances happen in the evening that are related to the maximum body temperature (2). On the other hand, iron metabolism is considered as one of the most challenging subjects in sports physiology (3). Iron is a vital mineral that plays a key role in the transfer of oxygen through hemoglobin $(\mathrm{Hb})$ to tissues, the storage of oxygen in the muscle by myoglobin, and numerous processes involved in the regeneration of oxidative adenosine triphosphate.
Further, the amount of iron in the tissues is one of the factors that determines the capacity of exercise in the individuals and is closely related to athlete performance (4). Football is an intense endurance-based sport that has a positive relationship with the reduction of iron levels, ferritin, and red blood cell (RBC). Several studies have focused on the effects of intense and strength training on the iron status of the body. The term "exercise-induced anemia" is often used to describe that $\mathrm{Hb}$ levels are close to clinical anemia (12 and $13 \mathrm{~g}$ of $\mathrm{Hb}$ per $100 \mathrm{~mL}$ in men and women, respectively). It is believed that this trouble is due to the intense exercise $(3,5,6)$. It is shown that the iron status and hematological indices of football players are undergoing significant changes as a result of exercise. Sporiš et al found that after a football match, the levels of iron and ferritin of under 21-year football players of the Croatian national team significantly reduced while there

(C) 2019 The Author(s); Published by Shahrekord University of Medical Sciences. This is an open-access article distributed under the terms of the Creative Commons Attribution License (http://creativecommons.org/licenses/by/4.0), which permits unrestricted use, distribution, and reproduction in any medium, provided the original work is properly cited. 
were no significant differences between the levels of RBC and $\mathrm{Hb}$ (6). Similarly, Silva et al observed a significant increase in the amount of $\mathrm{RBC}, \mathrm{Hb}$, and hematocrit after an intense anaerobic activity session (7). Jaksimovich et al showed that the 16-year-old football players of the Serbian national team had lower levels of 15 hematological indexes including $\mathrm{RBC}$, white blood cell, $\mathrm{Hb}$, ferritin, and iron during the tournament season and in the training sessions compared to non-athlete peers (8). It also seems that the duration, intensity, and the time of the activity and hence their effects on hematological parameters are important. Mohammad et al concluded that the iron surfaces, ferritin, and the RBC of professional Egyptian runners have an opposite relationship with the distance traveled by such runners (5). Based on the results of previous studies, iron seems to have a positive effect on sports activities and the performance of endurance activities such as football and delay fatigue $(9,10)$.

Obviously, RBC damage in the blood vessels of the leg is the reason for $\mathrm{Hb}$ and hematocrit decrease in athletes when they collide with the ground and damage the RBC in the capillary gastro-intestinal tract during exercise, especially long-term endurance exercise which may reduce the $\mathrm{Hb}$ concentration of the athletes (4). On the other hand, daytime rhythm regulates a large number of the cells, hormones, the cytokines of body, and the like, as well as the physiological functions of the cardiovascular system and blood. Therefore, more acute cardiovascular events occur in the morning (11). The philosophy of athletic training sessions in the morning and evening and at different times of the day is the reason for the importance of day-night rhythm. However, there is still no general agreement on the best exercise time. It is shown that blood pressure and heart beat rate demonstrate a day-night rhythm. In addition, the pattern of the day-night rhythm indicated the most severe cardiovascular events in the morning with regard to body temperature, blood pressure, blood fluidity, and fibrinogen changes $(9,12)$. Contradictory results are available about the effect of exercise time on hematological indices. Based on the result of previous research, plasma fibrinogen levels increased significantly after the morning exercise, but only a slight increase of $2 \%$ was observed in the afternoon (13). Further, it was found that the implementation of a session of maximal aerobic exercise in the morning and evening could have an effect on hematological factors, but the exercise time was unaffected by the changes (1). Furthermore, it was reported that the performance of professional women swimmers in the 100-meter was influenced by the time of the day and the subjects recorded a better time in the evening (2). Considering the above-mentioned issues and the importance of sports activities, especially the intense activities in the performance of professional athletes, the present research aimed to find whether there is a significant difference in the iron status and hematological factors of professional football players immediately after and 24 and 72 hours after acute aerobic activity in the morning and evening.

\section{Methods}

This study is a quasi-experimental single-blinded research with a repeated measure design. A total of 40 football players from Khuzestan clubs participated in this study. A nonprobability convenience sampling technique was used to collect the subjects. Moreover, they were selected from the same clubs and based on the same training, the lack of supplementation of vitamin C, iron, and B-group vitamins, adult group with no injuries and footballing history of at least 5 years. Regarding physiological differences, all subjects were selected from among the players, while no goalkeepers were used. The players came to visit exercise physiology lab and the Faculty of Physical Education in the Shahid Chamran University of Ahvaz (Iran) in order to become familiar with its environment. After they signed the letter of satisfaction for participation, anthropometric measurements including height, weight, and body mass index by bio-electrical impedance, and $\mathrm{VO}_{\text {max }}$ by Bruce test were conducted on a treadmill the Olympia 3/3, Javern South Korea (14). The subjects were assured that all their information would remain confidential. Additionally, the subjects were randomly assigned to four groups of morning exercises, morning control, evening exercise, and evening control (each containing $=10$ players). First, the football players were divided according to the defender, midfielder, or striker position and then randomly and equally from each post in each of the groups. The morning and evening exercise groups were performed the Bruce test respectively in the morning (8-10 AM) and evening (16-18 PM). Blood samples were taken before, immediately after, as well as 24 and 72 hours after the Bruce test in order to measure the serum iron levels, serum ferritin, RBC, and $\mathrm{Hb}$. Then, the samples were poured into the EDTA tubes (ethylene diamine tetra acetic acid) and transferred to the laboratory immediately after the completion of blood sampling. $\mathrm{RBC}$ and $\mathrm{Hb}$ in this study were measured by using an automatic counting machine (Mindray-BC 5300 Auto Hematology Analyzer). Next, the samples were centrifuged at a rate of $3500 \mathrm{rpm}$ for 10 minutes at $4^{\circ} \mathrm{C}$ to isolate the serum, followed by the extraction of the serum in special microtubes and storage at a temperature of $70^{\circ} \mathrm{C}$ until further measurement. Serum iron and ferritin were measured by ELISA method using a microplate reader (Hiperion Company, Germany) and by special kits (serum iron kit: Greiner manufactured in Germany, as well as ferritin kit: Pioneer Iranian Medicine Company). A 24-hour food questionnaire was developed to provide nutritional information to the subjects in order to record the food that they consume during the three days before and after the main test. The questionnaires were then analyzed by Nutritionist 4 software (15). For statistical 
analysis, the mean and standard deviation were used as descriptive statistics. Then, Shapiro-Wilkes test and Leven test were used to evaluate the normality of the data and the equality of variances, respectively. In addition, oneway ANOVA was utilized to compare the dietary data of all groups. Further, repeated-measures and two-way ANOVA (group*time) tests were applied for intra-group and between-group comparisons in each stage of the study. When the ANOVA test was significant, a post-hoc test was used for pairwise comparison. Data were analyzed by SPSS software, version 21 . The significance level was considered to be $P<0.05$.

\section{Results}

The mean and standard deviation of physiological and anthropometric characteristics of the subjects are presented in Table 1.

Furthermore, Table 2 shows the mean and standard deviation of the energy intake and nutrients status of the subjects in the four groups from 72 hours before and after the main test. Based on the results, there was no significant difference in the mean daily energy intake, the percentage of protein, carbohydrate, fat, fiber, vitamin $C$, vitamin B12, calcium, and iron among the four groups $(P \geq 0.05)$.

Similarly, Table 3 represents the levels of $\mathrm{RBC}, \mathrm{Hb}$, and the serum levels of iron and ferritin in four groups before and immediately after 24 and 72 hours after acute aerobic exercise. The results of two-way ANOVA demonstrated a significant difference regarding the effect of the interaction of group*time only on the serum levels of iron $(P=0.041)$.
Moreover, the results of ANOVA and Bonferroni post-hoc tests showed a significant increase in the $\mathrm{RBC}$ and $\mathrm{Hb}$ in the morning exercise group compared to the morning control group ( $P=0.001$ and $P=0.009$, respectively) and evening exercise group compared to the evening control group $(P=0.005$ and $P=0.013$, respectively). However, a significant decrease was observed in the serum levels of iron and ferritin in the morning training group compared to morning control $(P=0.008$ and $P=0.011$, respectively) and evening exercise groups compared to the evening control group $(P=0.003$ and $P=0.001$, respectively) immediately after the acute aerobic exercise. Additionally, there was a significant reduction immediately after, but a significant increase 24 hours after the acute aerobic exercise in the serum levels of iron in the morning exercise group compared to the evening exercise group ( $P=0.039, P=0.043$, respectively). As a result, comparing immediately and 24 hours after acute aerobic exercise, there was a significant increase in the serum levels of iron and ferritin in the morning exercise group versus morning control group $(P=0.022$ and $P=0.023$, respectively) and the evening exercise group compared to the control evening group $(P=0.007$ and $P=0.005$, respectively). This increase in the serum levels of iron in the exercise group was significant in the morning exercise group $(P=0.039)$. In addition, comparing immediately and 72 hours after the acute aerobic exercise, a significant reduction was found in the $\mathrm{RBC}$ and $\mathrm{Hb}$ concentration in the morning exercise group compared to morning control group $(P=0.02$ and $P=0.012$, respectively), as well as the evening exercise

Table 1. Anthropometric characteristics, as well as physical and physiological compositions of the subjects

\begin{tabular}{lllll}
\hline Group Factor & Morning Exercise & Evening Exercise & Morning Control & Evening Control \\
\hline Age $(\mathrm{y})$ & $22.6 \pm 5.6$ & $22.8 \pm 5.2$ & $23.1 \pm 5.4$ & $23.2 \pm 5.3$ \\
Height $(\mathrm{cm})$ & $177.3 \pm 6.7$ & $178.2 \pm 8.4$ & $178.7 \pm 7.5$ & $179.1 \pm 8.1$ \\
Weight $(\mathrm{kg})$ & $75.6 \pm 4.7$ & $77.4 \pm 7.3$ & $78.4 \pm 4.9$ & $77.2 \pm 5.1$ \\
BMI $\left(\mathrm{kg} / \mathrm{m}^{2}\right)$ & $24.1 \pm 1.5$ & $24.4 \pm 1.3$ & $24.2 \pm 2$ & $24 \pm 1.8$ \\
Fat percentage $(\%)$ & $15.9 \pm 1.2$ & $14.9 \pm 2.2$ & $15.1 \pm 1.8$ & $14.5 \pm 1.4$ \\
$\mathrm{Vo}_{\text {max }}(\mathrm{ml} / \mathrm{kg} / \mathrm{min})$ & $53.6 \pm 3.9$ & $54.8 \pm 4.1$ & $54.8 \pm 4.9$ & $55.1 \pm 4.8$ \\
\hline
\end{tabular}

Table 2. Comparison of the mean and standard deviation of nutrient and calorie intake 72 hours before and after the main test

\begin{tabular}{|c|c|c|c|c|c|}
\hline Factor & Morning Exercise & Evening Exercise & Morning Control & Evening Control & $P$ Value \\
\hline Energy (kcal/day) & $2993.2 \pm 218.1$ & $3116.6 \pm 197.4$ & $3018.8 \pm 209.5$ & $3110 \pm 213.6$ & 0.42 \\
\hline Protein (\%) & $18.3 \pm 2.4$ & $17.4 \pm 1.9$ & $18 \pm 2.1$ & $18.1 \pm 1.8$ & 0.61 \\
\hline Carbohydrate (\%) & $56.6 \pm 5.2$ & $54.4 \pm 4.9$ & $57.2 \pm 6.2$ & $56.4 \pm 5$ & 0.18 \\
\hline Fat $(\%)$ & $25.2 \pm 3.8$ & $29.8 \pm 3.4$ & $27.2 \pm 2.5$ & $26.2 \pm 3.6$ & 0.09 \\
\hline Fiber $(g / d)$ & $20.8 \pm 3.1$ & $22.1 \pm 5.6$ & $22.7 \pm 4.1$ & $21.3 \pm 4.1$ & 0.33 \\
\hline Vitamin C (mg/d) & $75.4 \pm 20.2$ & $73.3 \pm 17.9$ & $70.9 \pm 11.3$ & $72.6 \pm 12.2$ & 0.49 \\
\hline Vitamin B12 (mcg/d) & $2.8 \pm 1$ & $2.5 \pm 1.1$ & $2.4 \pm 0.9$ & $2.7 \pm 1.1$ & 0.27 \\
\hline Calcium (mg/d) & $452.3 \pm 170.3$ & $461.8 \pm 193.6$ & $448.4 \pm 162.4$ & $459.9 \pm 156.8$ & 0.53 \\
\hline Iron (mg/d) & $12.1 \pm 2.1$ & $11.4 \pm 2.5$ & $12.4 \pm 1.9$ & $12.6 \pm 2.1$ & 0.41 \\
\hline
\end{tabular}

Note. One-way ANOVA was used to compare the variables in four groups. The significance level was considered to be $\mathrm{P}<0.05$. 
Table 3. Comparison of the mean and standard deviation of serum RBC, $\mathrm{Hb}$, iron, and ferritin in subjects before, immediately after, 24 hours and 72 hours after acute aerobic exercise

\begin{tabular}{|c|c|c|c|c|c|c|}
\hline Factor & Group & Before Exercise & After Exercise & 24 h After Exercise & 72 h After Exercise & $P$-value (group*time) \\
\hline \multirow{4}{*}{$\begin{array}{l}\text { RBC } \\
\left(10^{3} / \mu \mathrm{L}\right)\end{array}$} & Morning exercise & $5178.8 \pm 187.5$ & $5449.6 \pm 279.1^{\mathrm{d}, \mathrm{a}}$ & $5399.6 \pm 213.2$ & $5202.2 \pm 302.2^{\mathrm{d}, \mathrm{c}}$ & \multirow{4}{*}{0.231} \\
\hline & Evening exercise & $5188.2 \pm 198.1$ & $5370.5 \pm 270.1^{\mathrm{e}, \mathrm{a}}$ & $5286.4 \pm 222.9$ & $5213.3 \pm 279.7^{\mathrm{e}, \mathrm{c}}$ & \\
\hline & Morning control & $5179.3 \pm 179.2$ & $5178.7 \pm 199.1$ & $5183.2 \pm 199.1$ & $5188.2 \pm 187.1$ & \\
\hline & Evening control & $5164.4 \pm 190$ & $5169.4 \pm 249.1$ & $5176.4 \pm 213.2$ & $5171.4 \pm 211.1$ & \\
\hline \multirow{4}{*}{$\mathrm{Hb}(\mathrm{g} / \mathrm{dL})$} & Morning exercise & $14.4 \pm 1.4$ & $15.1 \pm 1.7^{\mathrm{d}, \mathrm{a}}$ & $14.7 \pm 0.8$ & $14.4 \pm 1^{\mathrm{d}, \mathrm{c}}$ & \multirow{4}{*}{0.418} \\
\hline & Evening exercise & $14.4 \pm 1.7$ & $15 \pm 1^{e, a}$ & $14.7 \pm 0.7$ & $14.4 \pm 0.7^{\mathrm{e}, \mathrm{c}}$ & \\
\hline & Morning control & $14.5 \pm 1.6$ & $14.5 \pm 0.9$ & $14.4 \pm 1$ & $14.6 \pm 1.1$ & \\
\hline & Evening control & $14.2 \pm 1.1$ & $14.3 \pm 0.9$ & $14.2 \pm 0.9$ & $14.3 \pm 0.8$ & \\
\hline \multirow{4}{*}{$\begin{array}{l}\text { Iron } \\
(\mu \mathrm{g} / \mathrm{dL})\end{array}$} & Morning exercise & $112.5 \pm 4.3$ & $101.2 \pm 5.8^{\mathrm{f}, \mathrm{d}, \mathrm{a}}$ & $111.6 \pm 7.8^{f, d, b}$ & $114.8 \pm 6.1^{\mathrm{c}, \mathrm{d}}$ & \multirow{4}{*}{0.041} \\
\hline & Evening exercise & $113.5 \pm 3.5$ & $106.8 \pm 6.5^{e, a}$ & $112.3 \pm 6.1^{e, b}$ & $114.4 \pm 6^{\text {c,e }}$ & \\
\hline & Morning control & $112.1 \pm 6.1$ & $111.8 \pm 5.1$ & $111.1 \pm 4.2$ & $112.8 \pm 4.5$ & \\
\hline & Evening control & $112.8 \pm 4.1$ & $111 \pm 5.3$ & $111.3 \pm 4.2$ & $112.3 \pm 5.4$ & \\
\hline \multirow{4}{*}{$\begin{array}{l}\text { Frritin } \\
(\mathrm{ng} / \mathrm{mL})\end{array}$} & Morning exercise & $87.2 \pm 8.1$ & $79.2 \pm 10.8^{\mathrm{d}, \mathrm{a}}$ & $86.6 \pm 11.1^{\mathrm{d}, \mathrm{b}}$ & $86.8 \pm 11.8^{\mathrm{c}, \mathrm{d}}$ & \multirow{4}{*}{0.098} \\
\hline & Evening exercise & $86 \pm 7.9$ & $75.1 \pm 11.2^{\mathrm{e}, \mathrm{a}}$ & $84 \pm 11.2^{e, b}$ & $86.2 \pm 10.8^{\mathrm{c}, \mathrm{e}}$ & \\
\hline & Morning control & $86.1 \pm 48.3$ & $85.5 \pm 9.2$ & $86 \pm 8.4$ & $85.4 \pm 9.2$ & \\
\hline & Evening control & $88.9 \pm 8.2$ & $87.5 \pm 8.1$ & $86.5 \pm 9.1$ & $88.6 \pm 9.2$ & \\
\hline
\end{tabular}

Note. The results of repeated-measure ANOVA test for intra-group comparisons and one-way ANOVA for between-group comparisons at each stage of the test and two-way ANOVA (group*time) for the interaction of time and group.

The significance level was considered to be $P<0.05$.

a Significant difference between before exercise and immediately after the exercise.

b Significant difference between 24 hours and immediately after the exercise.

'Significant difference between 72 hours and immediately after the exercise.

dSignificant difference between morning exercise and morning control group.

e Significant difference between evening exercise and evening control group.

${ }^{\mathrm{f}}$ Significant difference between the morning exercise group and evening exercise group.

group compared to the evening control group $(P=0.013$ and $P=0.0018$, respectively). However, the serum levels of iron and ferritin demonstrated a significant increase in the morning exercise group compared to the morning control group ( $P=0.013$ and $P=0.021$, respectively), as well as the evening exercise group compared to the evening control group ( $P=0.002$ and $P=0.001$, respectively).

\section{Discussion}

Iron metabolism responded to sports activity as a challenge in the field of sports physiology and yielded contradictory results. The reduction, increase, and the stability of the iron levels are reported as a result of exercise activity (3). Further, the iron status and hematological parameters as a result of exercise activity depend on several factors such as the amount of iron excretion (16), RBC hemolysis $(17,18)$, intestinal absorption (19), andiron retention (20), along with the change and conversion of erythrocytes and $\mathrm{Hb}$ (21) or inflammation (22). A decrease in the levels of iron, ferritin, and $\mathrm{RBC}$ in intense exercise activity can be attributed to the hemolysis due to the stroke, which is related to the intensity of the exercise and the increase in temperature by $6 \%-11 \%$ excretion through sweating (3). Furthermore, the reduction of iron levels resulted from extreme sports activities is possibly due to a decline in the transfer of bivalent metal (Divalent metal transporter-1),
Ferroportin 1, the carrier protein (Haem carrier protein-1), hephaestin, and ceruloplasmin $(9,19)$.

The results of this study showed that RBC and $\mathrm{Hb}$ levels in the morning and evening exercise groups were significantly higher than those in the control groups after the aerobic activity, while the iron and ferritin levels decreased significantly. Moreover, the reduction in the serum levels of the iron in the evening training group was significant compared to the morning training group. Regardless of the exercise time, a significant increase was observed in $\mathrm{Hb}$ and $\mathrm{RBC}$ in both training groups. Our research results are consistent with those of many studies on hematological profiles. Significant increases in $\mathrm{Hb}$ and RBC in teenage Serbian football players were reported in relation to non-athlete peers (8). Additionally, in line with the current study, Mohamed et al, in their study on female professional runners in Egypt (short distance runners $(n=6)$, middle distance runners $(n=5)$, and long distance runners $(n=3))$, found an inverse proportional relationship between iron and ferritin levels and the distance of running among elite runners so that the iron levels decrease by increasing the running distance (5). In addition, Sporiš et al showed that the levels of iron and ferritin of men football players in the under-21 Croatian team significantly decreased after a football match. They also reported a non-significant reduction in $\mathrm{RBC}$ and $\mathrm{Hb}$ 
levels (6). Regarding the effect of exercise time, the result of a study revealed that a session of intense aerobic activity in the morning and evening increased the RBC, white blood cells, and $\mathrm{Hb}$, but no difference was observed in this regard between the two times (1). On the other hand, fibrinogen levels are found to increase after the endurance and resistance training in the morning. Therefore, from the point of view of cardiovascular health, exercise is safer in the evening (3). Based on the available information, exercise activity causes erythrocytosis, which increases the concentration of erythrocytes to $25 \%$. These changes are initially justified by the call for blood supply because the blood stores a large number of cells, but less plasma compared to the circulating blood (23). Iron is also used in the bone marrow, liver, and spleen, and ferritin is utilized to reconstruct $\mathrm{Hb}$ and $\mathrm{RBC}$, to maintain high levels of $\mathrm{RBC}$ and $\mathrm{Hb}$, and to transport the oxygen to the tissues (3). Therefore, given the intensity of exercise, the increase in $\mathrm{Hb}$ and $\mathrm{RBC}$ and the reduction in iron and ferritin in the present study are such that the increase in temperature, sweating, inflammation, decreased plasma volume, and the destruction of the blood cells can decrease the levels of hematological indices and are used to maintain the levels of $\mathrm{Hb}$ and $\mathrm{RBC}$, iron, and ferritin in the liver and spleen (23). The results of this study further showed that the iron levels and serum ferritin in the morning and evening exercises group increased significantly after 24 hours of recovery following the exercise activity in comparison with the control group. Furthermore, the serum iron levels in the evening exercise group increased significantly compared to the morning exercise group. Conversely, 72 hours of recovery after the acute aerobic activity significantly decreased the levels of $\mathrm{Hb}$ and $\mathrm{RBC}$ while a significant increase was observed in serum iron and ferritin levels in the morning and evening exercise groups compared to those in the morning and evening control groups. There is not much research in the field of changes in hematology factors and iron indices during recovery. Accordingly, 2 hours of recovery after the acute aerobic exercise was reported to have no significant effect on $\mathrm{Hb}$ and $\mathrm{RBC}$ of the athlete men (1). However, no significant changes in fibrinogen levels and high sensitivity C-reactive protein were found in active men three hours after the endurance and resistance exercise (3). Therefore, it seems that more time is needed for recovery and changes in hematological factors after the exercise, especially severe physical activity and the levels of blood factors and plasma volume re suggested to return to their initial level after 48-72 hours of acute aerobic exercise (24). Sweating is argued to be one of the cases that reduce the iron levels during intense activities and excrete between $6 \%$ and $11 \%$ of body iron (25). Iron retention is regarded as another iron-depleting cause after the exercise, which is called "exercise-induced anemia". An increase in plasma volume due to intracellular fluid transfer to the vessel and the reduction of $\mathrm{Hb}$ concentration are believed to lead to such anemia. Predictably, the increase in plasma volume that occurs as a result of the training is initiated 48-72 hours after the exercise (24). In the present study, iron and ferritin levels increased after 24 and 72 hours of recovery. Based on the results of a study, an exercise in the morning caused a greater reduction in the serum levels compared to the evening exercise, which could be due to higher temperatures and consequently sweating (26). Contrarily, after 24 hours of recovery, a greater increase in the serum iron of the morning training group was observed compared to the evening exercise group, which is likely to be in line with other changes as a result of alterations in plasma volume (20). In our study, a decrease in $\mathrm{Hb}$ and $\mathrm{RBC}$ was observed 72 hours after the acute aerobic exercise. However, a significant increase was found in $\mathrm{RBC}$ and $\mathrm{Hb}$ levels during acute aerobic activity as well. At first, the mechanism of this increase and decrease probably demonstrates negative feedback during the intensive training, which is due to the need for more oxygen. Moreover, the synthesis of $\mathrm{Hb}$ and RBC increases while the synthesis of RBC cells decreases as the level of erythropoietin secretion decreases (27). Therefore, iron intake for the synthesis of $\mathrm{Hb}$ is considered as one of the reasons for a reduction in the serum levels of iron and ferritin. In the recovery period, the levels of $\mathrm{Hb}$ and $\mathrm{RBC}$ decrease and reach their initial levels with changes in plasma volume, the levels of erythropoietin, and the oxygen demand (9).

\section{Conclusion}

Regarding the results of this study, it seems that performing the exercise activity in the evening is important in terms of the reduction of serum iron levels and the occurrence of "exercise-induced anemia". On the other hand, according to the results of the present study, professional football players require at least 24-72 hours to recover after intensive endurance exercises in order to restore safe levels of hematological parameters.

Conflict of interests

None.

Ethical considerations

All research stages were approved by the Ethics Committee of Shahid Chamran University of Ahvaz (EE/98.24.3.77899/scu.ac.ir).

\section{Acknowledgments}

This article, as part of an approved project at Shahid Chamran University of Ahvaz, received the grant (No. 862) and was financially supported by the Vice-chancellor for Research Affairs, Shahid Chamran University of Ahvaz. Therefore, the authors would like to express their gratitude to the subjects of this research, as well as the coaches and club management for their unmistakable cooperation for implementing this study.

\section{References}

1. Mohammad Najad Panah Kandi Y, Mohammad Najad Panah Kandi A, Shahidi F, Masoudian B. The effect of a maximal aerobic exercise session in the morning and afternoon on 
certain hematological factors in young athletes. Razi Journal of Medical Sciences. 2013;20(106):20-9.

2. Rahnama N, Bambaeichi E, Sadeghipoor HR. Effect of circadian rhythm on the performance and some physiological factors of young boy swimmers. Journal of Movement Science \& Sports. 2008;6(12):67-76.

3. Lashkari F, Samavati Sharif MA, Ranjbar K. The effect of two different modes of exercise swimming and vitamin $C$ supplementation on anemia indices in male wistar rat. Journal of Knowledge \& Health. 2015;11(1):55-61. doi: 10.22100/jkh. v11i1.1138.

4. Rahnama N. Exercise biochemistry. Tehran: Samt; 2010. [Persian].

5. Elgushy M, Mohamed EE. Iron status for the egyptian female players in running competition (short-middle-long distances)comparative study. Ovidius Univ Ann Ser Phys Educ Sport Sci Mov Health. 2016;16(2 Suppl): 449-453.

6. Sporis G, Vlahovic T, Trajkovic N, Milanovic Z, Madic D. Haematological and iron status following a soccer match. Facta Univ Phys Educ Sport. 2016;14(2):289-95.

7. Silva AS, Santhiago V, Papoti M, Gobatto CA. Hematological parameters and anaerobic threshold in Brazilian soccer players throughout a training program. Int J Lab Hematol. 2008;30(2):158-66. doi: 10.1111/j.1751-553X.2007.00919.x.

8. Joksimovic A, Stankovic D, Ilic D, Joksimovic I, Jerkan M. Hematological profile of Serbian youth national soccer teams. J Hum Kinet. 2009;22:51-9.

9. Lane DJ, Richardson DR. The active role of vitamin C in mammalian iron metabolism: much more than just enhanced iron absorption! Free Radic Biol Med. 2014;75:69-83. doi: 10.1016/j.freeradbiomed.2014.07.007.

10. Bourque SP, Pate RR, Branch JD. Twelve weeks of endurance exercise training does not affect iron status measures in women. J Am Diet Assoc. 1997;97(10):1116-21. doi: 10.1016/ s0002-8223(97)00272-1.

11. Rudnicka AR, Rumley A, Lowe GD, Strachan DP. Diurnal, seasonal, and blood-processing patterns in levels of circulating fibrinogen, fibrin D-dimer, C-reactive protein, tissue plasminogen activator, and von Willebrand factor in a 45-year-old population. Circulation. 2007;115(8):996-1003. doi: $\quad$ 10.1161/circulationaha.106.635169.

12. Ahmadizad S, Bassami M. Interaction effects of time of day and sub-maximal treadmill exercise on the main determinants of blood fluidity. Clin Hemorheol Microcirc. 2010;45(24):177-84. doi: 10.3233/ch-2010-1295.

13. Kousari E, Mousavi SM, Rawasi AA, Akbarnejad A, Kousari Z, Kousari $\mathrm{H}$. Comparison of exercise performance at two times in the morning and afternoon on cardiovascular indicators in active men's. Res Physiol Manag Sports. 2014;6(2):21-34.

14. Khodadadi H, Rajabi H, Attarzadeh SR, Abbasian S. The effect of high intensity interval training (HIIT) and pilates on levels of irisin and insulin resistance in overweight women. Iranian Journal of Endocrinology and Metabolism. 2014;16(3):190-6.

15. Shirinzadeh $M$, Shakerhoseini R, Hoshyarrad A. Nutrient value and adequacy of consumed meal in patient with type II diabetes. Iranian Journal of Endocrinology and Metabolism. 2009;11(1):25-32.

16. Maughan RJ. Role of micronutrients in sport and physical activity. Br Med Bull. 1999;55(3):683-90. doi: 10.1258/0007142991902556.

17. Skarpanska-Stejnborn A, Basta P, Trzeciak J, SzczesniakPilaczynska L. Effect of intense physical exercise on hepcidin levels and selected parameters of iron metabolism in rowing athletes. Eur J Appl Physiol. 2015;115(2):345-51. doi: 10.1007/ s00421-014-3018-3.

18. Yusof A, Leithauser RM, Roth HJ, Finkernagel H, Wilson MT, Beneke R. Exercise-induced hemolysis is caused by protein modification and most evident during the early phase of an ultraendurance race. J Appl Physiol (1985). 2007;102(2):5826. doi: 10.1152/japplphysiol.00580.2006.

19. Fujii T, Matsuo T, Okamura K. Effects of resistance exercise on iron absorption and balance in iron-deficient rats. Biol Trace Elem Res. 2014;161(1):101-6. doi: 10.1007/s12011-0140075-8.

20. Waller MF, Haymes EM. The effects of heat and exercise on sweat iron loss. Med Sci Sports Exerc. 1996;28(2):197-203.

21. Inoue $Y$, Matsui A, Asai Y, Aoki F, Matsui T, Yano H. Effect of exercise on iron metabolism in horses. Biol Trace Elem Res. 2005;107(1):33-42. doi: 10.1385/bter:107:1:033.

22. Sim M, Dawson B, Landers G, Swinkels DW, Tjalsma H, Trinder D, et al. Effect of exercise modality and intensity on post-exercise interleukin-6 and hepcidin levels. Int J Sport Nutr Exerc Metab. 2013;23(2):178-86.

23. Bobeuf F, Labonte M, Khalil A, Dionne IJ. Effect of resistance training on hematological blood markers in older men and women: a pilot study. Curr Gerontol Geriatr Res. 2009:156820. doi: 10.1155/2009/156820.

24. Adams WD, Wild F, Hill RMG. Hematologic Concerns in the Runner. In: Test book of Running Medicine. McGraw-Hill; 2001.

25. Liu YQ, Chang YZ, Zhao B, Wang HT, Duan XL. Does hepatic hepcidin play an important role in exercise-associated anemia in rats? Int J Sport Nutr Exerc Metab. 2011;21(1):19-26.

26. Ming Qian Z, Sheng Xiao D, Kui Liao Q, Ping Ho K. Effect of different durations of exercise on transferrin-bound iron uptake by rat erythroblast. J Nutr Biochem. 2002;13(1):47-54.

27. Neilands JB. Microbial Iron metabolism: A comprehensive treatise. 1st ed. United States: Academic Press; 2014:319-20. 